\title{
La formación inicial de la competencia profesional específica registrar hechos económicos mediante la Contabilidad General.
}

\section{The initial training of the specific professional competence to record economic events through General Accounting.}

\author{
Elizabet Prado Chaviano. ${ }^{1}$, Pilarín Baujín Pérez. ${ }^{2}$, Margarita González González. ${ }^{3}$ \& \\ Maribel del Rocío Paredes Cabezas. ${ }^{4}$
}

\begin{abstract}
DOI: https://doi.org/10.33262/concienciadigital.v4i2.1.1706

The study presented responds to a four-year scientific investigation and addresses the initial training of the specific professional competence to record economic facts, which has a complex and systemic nature, where knowledge, skills, values, motivations, and performances, based on the aspects of the recording of economic events in the teachinglearning process of General Accounting I, II, III and IV subjects, which have repercussions on Accounting and Financial Labor Practice I and in other contexts of action. A diagnosis was made for which methods of the empirical level are used, such as: observation, survey and document review, which evidenced the need to develop a procedure for the initial formation of specific professional competence to record economic facts, For this, a set of stages and steps were articulated and the transformations that occurred with the preparation and implementation of theoretical, practical and integrative exercises, the Arcont software, case studies, accounting procedures, activities,

\footnotetext{
${ }^{1}$ MSc en Gestión Turística y doctorando en Ciencias de la Educación Profesora del Dpto de Contabilidad y Finanzas. Universidad de Matanzas. Cuba. Correo electrónico: elizabetpradochaviano7979@ gmail.com ORCID:0000-0002-5388-081X

${ }^{2}$ Contables y Financieras. Profesora del departamento de Contabilidad y Finanzas. ORCID:0000-00020984-3368 Correo electrónico: pilarin.baujin@umcc.cu ORCID:0000-0002-5388-081X

3 Pedagógicas. Profesora investigadora del Centro de Estudios Educativos. Universidad de Matanzas. Cuba. Correo electrónico: gonzalezgonzalez.margarita4@gmail.com ORCID: orcid.org/0000-00023041-6387.

4 Universidad Técnica de Ambato, Facultad de contabilidad y auditoria, Ecuador, maribelparedes@uta.edu.ec, https://orcid.org/0000-0002-8449-5404.
} 
as well as the methodological classes, visits to the Accounting department and the improvement of Accounting and Financial Practice I. Keywords: professional skills, skills training, recording economic facts, interdisciplinarity.

Keywords: professional skills, skills training, recording economic events, interdisciplinarity.

\section{Resumen:}

El estudio que se presenta responde a una investigación científica de cuatro años de ejecución y aborda la formación inicial de la competencia profesional específica registrar hechos económicos, la cual tiene un carácter complejo y sistémico, donde se integran conocimientos, habilidades, valores, motivaciones, y desempeños, a partir de los aspectos del registro de hechos económicos en el proceso de enseñanza aprendizaje de las asignaturas Contabilidad General I, II, III y IV, lo que repercuten en la Práctica Laboral Contable y Financiera I y en otros contextos de actuación. Se realizó un diagnóstico para lo cual se utilizan métodos del nivel empírico, tales como: la observación, la encuesta y la revisión de documentos, el cual evidenció la necesidad de elaborar un procedimiento para la formación inicial de la competencia profesional específica registrar hechos económicos, para ello se articularon un conjunto de etapas y pasos y se constataron las transformaciones ocurridas con la elaboración e implementación de ejercicios teóricos, prácticos e integradores, el software Arcont, casos de estudio, procedimientos contables, actividades, así como las clases metodológicas, las visitas al departamento de Contabilidad y el perfeccionamiento de la Práctica Contable y Financiera I.

Palabras claves: competencias profesionales, formación de competencias, registrar hechos económicos, interdisciplinariedad.

\section{Introducción:}

En los estudios analizados sobre la formación de competencias profesionales en el entorno educativo, se destacan autores como (Ramos, 2017 y Pérez, 2019) que realizan investigaciones desde las asignaturas y las carreras, en las cuales se evidencia el perfeccionamiento del concepto de competencias profesionales en cuanto a su rigor, profundidad y amplitud, donde no solo están presentes los conocimientos y habilidades en el ejercicio de una profesión, sino también se refiere a los modos de actuación en el entorno socioeconómico en el que desempeña su función profesional.

El estudio que se presenta, estuvo precedido de investigaciones sobre la competencia profesional específica registrar hechos económicos donde se destaca (SINEACE, 2014), que solo se señala algunas tareas a desarrollar, pruebas y criterios de desempeño vinculados a la preparación de los asientos de diarios en los libros obligatorios y auxiliares, según plan contable, las normas legales vigentes, que incluye, la verificación y conciliación de los hechos económicos, pero no fundamenta esta competencia desde el 
punto de vista teórico, ni estructuran las posibles vías de formación en el proceso de enseñanza aprendizaje.

Esta investigación incluye la vinculación de forma holística de los componentes del contenido de enseñanza, la motivación y el desempeño, lo cual está vinculado a las exigencias del Plan de Estudio E y en correspondencia con las normas cubanas de contabilidad y las normas internacionales de la información financiera, así como las técnicas que rigen el proceso contable (MES, 2017) y su presencia dentro de los objetivos generales de la disciplina Contabilidad, en aras de contribuir a la formación de un profesional "capaz de registrar, presentar y analizar diferentes hechos económicos vinculados a la práctica contable, en correspondencia con los cambios que se llevan a cabo en el país" (MES, 2017, p. 66). En este sentido se presenta un procedimiento para la formación inicial de la competencia profesional específica registrar hechos económicos en los estudiantes, mediante el proceso de enseñanza aprendizaje de las asignaturas Contabilidad General I, II, III y IV de la carrera Contabilidad y Finanzas en la Universidad de Matanzas.

A nivel internacional se refieren estudios de las competencias profesionales en la carrera Contabilidad y Finanzas, en Europa se han trabajado las competencias, por ejemplo: en la de Málaga (UMA, 2019) donde se diseñan programas para la formación de competencias profesionales integradas al proyecto Tuning, mientras que en las universidades cubanas aunque los planes de estudio están diseñados en función del desarrollo de habilidades profesionales (MES, 2015 y 2017), comienzan a presentarse investigaciones sobre las competencias profesionales generales y específicas en la carrera Contabilidad y Finanzas (Tobar, et al., 2018) y Pérez, 2019).

En el caso de la competencia profesional específica registrar hechos económicos se ha reconocido en otros entornos, como por ejemplo, en Perú (SINEACE 2014), pero este sistema se limita a la confección de perfiles laborales para los especialistas de Gestión Económica Financiera, también el Sistema Nacional de Aprendizaje de Colombia SENA (2017) trata el registro de hechos económicos, como una competencia profesional específica mínima a desarrollar, pero no hace alusión a la tipificación de los hechos económicos y las regularidades de las cuentas del registro de los hechos económicos.

Los autores referenciados que identifican el registro como una competencia profesional específica en la carrera Contabilidad y Finanzas, lo enfocan desde el desempeño laboral en el puesto de trabajo y desde la formación académica en el proceso de enseñanza aprendizaje, por lo que no conciben esta competencia profesional específica como la integración de conocimientos, habilidades, valores, motivación y desempeño donde intervienen los profesores, tutores, estudiantes y otros contextos de actuación.

El análisis realizado le permitió a los autores considerar la competencia profesional específica registrar hechos económicos, como un proceso continuo, complejo y sistémico que integra conocimientos, habilidades, valores éticos del contador, motivaciones y desempeños relacionados con el análisis y procesamiento de los hechos económicos para 
su posterior registro acorde con la normativa contable evidenciados en la actividad académica y el entorno laboral con la ayuda del profesor, el tutor y otros contextos de actuación.

La formación inicial de esta competencia ocurre en los dos primeros años mediante el proceso de enseñanza de la disciplina Contabilidad, donde se registran los hechos económicos en diversas entidades y se desarrollan en el estudiante los modos de actuación profesional. En este proceso se manifiestan las relaciones de precedencia con las asignaturas Contabilidad General y con las demás disciplinas, y se profundiza en el registro de los hechos económicos de la Contabilidad Gerencial en el entorno académico y laboral. En la formación inicial se tienen en cuenta los sistemas de conocimientos, tales como, la normativa contable, la documentación mercantil, las cuentas y subcuentas, los hechos económicos, el método de la Partida Doble así como los procedimientos contables. Asimismo, deben contemplarse los valores éticos del contador que se materializan en el correcto registro de los bienes y recursos que poseen las entidades, el cumplimiento de las normas internacionales y cubanas y la colaboración mediante el trabajo en equipo en el proceso de enseñanza y con los especialistas en el área contable en el entorno laboral y en otros contextos de actuación.

Otro aspecto a tener en cuenta es la motivación, en lo que desempeña un papel importante las relaciones interpersonales que se ponen de manifiesto en el trabajo colectivo y en el interés por realizar las tareas. Esto contribuye a crear la posibilidad de determinar las limitaciones y logros al registrar los hechos económicos en la actividad académica y laboral y por consiguiente a demostrar el desempeño alcanzado.

A partir de los elementos analizados se define la formación inicial de esta competencia como un proceso continuo con un carácter complejo y sistémico, que se inicia en el proceso de enseñanza aprendizaje de la Contabilidad General, durante el primero y segundo años de la carrera Contabilidad y Finanzas, que integra conocimientos, habilidades, valores éticos del contador, motivaciones, y desempeños a partir de los aspectos del registro de hechos económicos y se profundiza y sistematiza mediante la ejercitación del propio registro en diversas entidades, lo que permite al estudiante identificar las limitaciones y progresos en el aprendizaje, tanto en el entorno académico como en el laboral y en otros contextos de actuación, con la ayuda del profesor y el tutor.

\section{Metodología.}

Para la formación inicial de la competencia profesional específica registrar hechos económicos, se presenta a continuación un procedimiento donde se describen los pasos y etapas a desarrollar en la disciplina Contabilidad General en la carrera Contabilidad y Finanzas.

Etapa 1. Diagnóstico: se caracterizó la preparación con respecto a la formación inicial de la competencia profesional específica registrar hechos económicos y se recomiendan los pasos siguientes: 
- Paso 1. Presentación de la estructura y concepción del procedimiento al colectivo de la disciplina Contabilidad.

- Paso 2. Identificación de las dificultades y avances en el tratamiento teórico, didáctico y práctico del registro de los hechos económicos, los valores éticos del contador y la motivación, el desempeño, así como la identificación de las limitaciones y progresos en el aprendizaje en las asignaturas de Contabilidad General, la Práctica Laboral Contable y Financiera I y otros contextos de actuación.

Etapa 2. Planificación-ejecución: Se realizó la planificación y ejecución de los pasos para la formación inicial de la competencia profesional específica registrar hechos económicos en las asignaturas de Contabilidad General

- Paso 1. Planificación y ejecución de clases metodológicas para la preparación teórica y didáctica de los profesores.

- Paso 2. Preparación e implementación de un caso práctico en la asignatura Contabilidad General II.

- Paso 3. Confección e implementación de ejercicios, videos, procedimientos contables, la visita al departamento de Contabilidad y el sistema de evaluación para afianzar los conocimientos teóricos y prácticos del registro de los hechos económicos, en las asignaturas Contabilidad General I, II, III y IV.

- Paso 4. Planificación y ejecución de un taller, un ejercicio integrador y la Práctica Laboral Contable y Financiera I y tareas a desarrollar en otros contextos de actuación.

- Paso 5. Preparación del contenido sobre la fiscalización de los documentos mercantiles, procedimientos contables, los hechos económicos y su tipificación y las regularidades de las cuentas en el registro de los hechos económicos en diversas entidades.

Etapa 4. Evaluación de los resultados de los pasos ejecutados, para valorar como ellos contribuyen a la formación inicial de la competencia profesional específica registrar hechos económicos mediante la valoración de la efectividad de las acciones desarrolladas en la ejecución del procedimiento.

\section{Resultados.}

El procedimiento se introdujo en el primer año del curso 2018-2019, en este caso con 65 estudiantes en el curso 2019-2020, en las asignaturas de Contabilidad General.

\section{Etapa 1. Diagnóstico}

Paso 1. Presentación de la estructura y concepción del procedimiento al colectivo de la disciplina Contabilidad. Se realizó un taller donde se presentó la estructura y concepción del procedimiento, estuvieron presentes la coordinadora de la carrera, el jefe del departamento y los profesores del colectivo de la disciplina Contabilidad. 
Paso 2. Identificación de las dificultades y avances en el tratamiento teórico, didáctico y práctico del registro de los hechos económicos, los valores éticos del contador y la motivación, el desempeño, así como la identificación de las limitaciones y progresos en el aprendizaje en las asignaturas de Contabilidad General, la Práctica Laboral Contable y Financiera I y otros contextos de actuación. Las fortalezas relacionadas con el registro de los hechos económicos se centran en la declaración de los contenidos, objetivos, medios y métodos en las clases y algunos conocimientos teóricos relacionados con las entidades, las cuentas, el método de la Partida Doble, la normativa contable y los libros obligatorios de la contabilidad y las limitaciones que se aprecian son la falta de integración los conocimientos, habilidades, valores éticos del contador, motivación y desempeños en las conferencias, clases prácticas de las asignaturas mencionadas, otros contextos de actuación y en la Práctica Laboral Contable y Financiera I y el escaso uso de los nexos interdisciplinarios con las demás asignaturas de los dos primeros años

\section{Etapa 2. Planificación-ejecución}

Paso 1. Planificación y ejecución de clases metodológicas para la preparación teórica y didáctica de los profesores. En este sentido, se planificaron y ejecutaron tres clases metodológicas sobre el uso de medios y métodos de enseñanza y diferentes software que se utilizan para el registro de los hechos económicos donde se realiza un intercambio al final con los profesores.

Paso 2. Preparación e implementación de un caso práctico en la asignatura Contabilidad General II. La totalidad de los profesores, tutores y estudiantes señalaron la importancia de los casos prácticos sobre el registro de los hechos económicos en diferentes tipos de entidades con un enfoque interdisciplinario, resueltos de forma manual y en software.

Paso 3. Confección e implementación de ejercicios, videos, procedimientos contables, la visita al departamento de Contabilidad y sistema de evaluación para afianzar los conocimientos teóricos y prácticos del registro de los hechos económicos, en las asignaturas de Contabilidad General, para lo cual se reunieron los profesores participantes y se aplicó la técnica de tormenta de ideas, donde el $100 \%$ de los profesores evaluaron como importantes las actividades, los videos, procedimientos contables, los ejercicios teóricos y prácticos y la visita al departamento contable para afianzar los conocimientos relacionados con la formación inicial de esta competencia profesional.

Paso 4. Planificación y ejecución de un taller, un ejercicio integrador y la Práctica Laboral Contable y Financiera I y tareas a desarrollar en otros contextos de actuación. Se consideró en su evaluación en sentido general, la integración de conocimientos, habilidades, motivación, valores y desempeños, así como las relaciones interdisciplinarias en los dos años y el $84.61 \%$ (55) estudiantes integraron los aspectos para la formación inicial de esta competencia profesional y el resto, (10) estudiantes, que representan el $15.38 \%$, solo integraron los aspectos relacionados con las entidades, los hechos económicos y la normativa contable. En el ejercicio integrador todos los estudiantes aplicaron los contenidos de las demás asignaturas del año, se realiza en equipos de dos 
participantes, donde el $64.61 \%$ (42) estudiantes entregaron todas las tareas según el cronograma establecido y mostraron un buen desempeño en la formación inicial de esta competencia profesional específica, el resto presentó algunas limitaciones, esencialmente en los hechos económicos, relacionados con las situaciones excepcionales y la motivación por mejorar la calidad en la presentación del ejercicio.

En la Práctica Laboral Contable y Financiera I, todos los estudiantes presentaron un aval de la entidad donde realizaron las prácticas y un anexo con un ejercicio del registro de los hechos económicos, para ello, se apoyaron en los conocimientos de las asignaturas de Contabilidad General y Contabilidad de Gestión I. En este caso, el 40 \% (26) estudiantes, dieron criterios positivos del trabajo de los compañeros, aunque el 30.76\% (20) estudiantes, lograron señalar aspectos que debían ser profundizados como el registro más detallado de los hechos económicos, porque en los casos presentados solo se tuvieron en consideración las cuentas y el 29.23\% (19) estudiantes no emitieron ningún criterio.

En la evaluación de otros contextos de actuación se consideró positiva la forma en que se evaluó su ejecución y aunque el 100\% de los profesores supervisó las tareas de apoyo a las entidades territoriales y los estudiantes reconocieron la oportunidad de constatar la teoría estudiada sobre los inventarios en cuanto a los métodos utilizados y documentos mercantiles así como el control de los Activos fijos Tangibles.

Paso 5. Preparación del contenido sobre la fiscalización de los documentos mercantiles, procedimientos contables, los hechos económicos y su tipificación y las regularidades de las cuentas en el registro de los hechos económicos en diversas entidades. Todos los profesores, tutores y estudiantes consideraron como positivo la planificación e incorporación en las asignaturas de Contabilidad General de estos contenidos relacionados con los hechos económicos.

\section{Etapa 3. Evaluación}

Paso 1. Valoración de la efectividad de las acciones de la estrategia didáctica. Se midió la efectividad a través de un grupo focal compuesto por siete profesores de las disciplinas del perfil profesional y los 15 tutores que atienden la Práctica Laboral Contable y Financiera I. En este caso, los criterios sobre la estrategia fueron positivos, teniendo en cuenta que las acciones tributan a la formación inicial de esta competencia profesional.

Además se aplicó un test de comprobación a los estudiantes al concluir el primer semestre del segundo año en el curso 2019-2020 para medir la formación inicial de esta competencia profesional específica al terminar la asignatura Contabilidad General IV, donde de los 65 estudiantes el 15.38\% (10) estudiantes, dominan los procedimientos contables y se apoyan en ellos al registrar hechos económicos de forma manual y/o software, así como los libros obligatorios de la contabilidad y la tipificación de los hechos económicos. El 60\% (39) estudiantes, conocen las cuentas que intervienen en el registro de los hechos económicos en entidades industriales, comerciales y se servicio así como la ubicación de los productos en el almacén y respectivamente el llenado de la documentación, saben tipificar los hechos económicos y se establecen las relaciones entre 
las asignaturas de Contabilidad General, Contabilidad de Gestión I y Sistema Financiero, mientras que el resto de los estudiantes solo lo conocen en entidades comerciales. El $24.61 \%$ (16) estudiantes, conocen cuál es el destino de los documentos mercantiles, y su tratamiento en términos de fiscalización; así como los cambios a realizar en cuanto a la nomenclatura de las cuentas para diferentes entidades, cómo comprobar la exactitud, prudencia y confiabilidad al registrar hechos económicos.

La asistencia y responsabilidad puso de manifiesto la motivación de los estudiantes por su formación profesional, así como las limitaciones que aún se mantienen y los progresos alcanzados con la aplicación del procedimiento.

\section{Conclusiones}

- El análisis realizado de los referentes teóricos permitió reconocer el registro de hechos económicos como una competencia profesional del licenciado en Contabilidad y Finanzas y definir la variable formación inicial de la competencia profesional específica registrar hechos económicos. La caracterización del estado actual de los estudiantes en la formación inicial de la competencia señalada, puso de manifiesto dificultades en los aspectos diagnosticados, por lo cual, se estructuró y se puso en práctica un procedimiento dirigido a la preparación de profesores y tutores y al trabajo desarrollado por los estudiantes, con tres etapas. El cual puso de manifiesto transformaciones cualitativamente superiores en la carrera Licenciatura en Contabilidad y Finanzas de la Universidad de Matanzas, en relación a la formación inicial de la competencia profesional registrar hechos económicos.

\section{Referencias bibliográficas:}

Capó, J. R., Castillo, J. D y González, S., (2013). Sistema de gestión integrada de capital humano para las universidades cubanas. Caso Universidad Agraria de La Habana. Revista Congreso Universidad, II (3). Habana, Cuba: Editorial Félix Varela.

Martínez, M., Álvarez de Eulate, C. Y., y Villardón, L. (2017). Estudos e desenvolvimento das competências profissionais. Revista Iberoamericana de Educación. 74(1), p.171-192. Recuperado de: http//dialnet.uniroja.es/ejemplar/461813.

MES. (2015). Documento base para la elaboración del Plan de Estudio E. La Habana (Cuba).

Pérez, T. (2019). La formación inicial de la competencia profesional resolver problemas económicos mediante el proceso de enseñanza aprendizaje de la Econometría (Tesis doctoral, Universidad de Matanzas Sede Juan Marinello, Matanzas, Cuba).

Ramos, O. (2017). Estrategia educativa para el desarrollo de la competencia preventiva del equipo de salud bucal en las enfermedades bucodentales (Tesis doctoral en 
Ciencias Pedagógicas, Universidad de Ciencias Pedagógicas Enrique José Varona. La Habana, Cuba).

SENA. (2017). Sistema Nacional de aprendizaje. Bogotá. Cundinamarca. Colombia. Recuperado de: http://sena.edu.cu.

SINEACE. (2014). Sistema Nacional de Evaluación, Acreditación y Certificación de la Calidad de la Educación. Normas de competencia del profesional técnico en Contabilidad. Recuperado de: http//cir@sineace.gob.pe/www.sineace.gob.pe

Tobar, G., Arias, M., y Ríos, G. (2018). Competencias del contador-auditor en el perfil de egreso. Revista de Actualidad Contable Faces, 21 (37), p.2-56. Recuperado de: http//:www.redalyc.org/artículo.oa?id=25755483005.

UMA. (2019). Universidad de Málaga. España. Grado en Finanzas y Contabilidad. Recuperado de: https://www.uma.es/grado-en-finanzas-ycontabilidad/info/8957/competencias-finanzas-y-contabilidad.

\section{Ciencia}




\section{PARA CITAR EL ARTÍCULO INDEXADO.}

Prado Chaviano, E., Baujín Pérez, P., González González, M., \& Paredes Cabezas, M. del R. (2021). La formación inicial de la competencia profesional específica registrar hechos económicos mediante la Contabilidad General. ConcienciaDigital, 4(2.1), 52-61. https://doi.org/10.33262/concienciadigital.v4i2.1.1706

\section{LCiencia}

El artículo que se publica es de exclusiva responsabilidad de los autores y no necesariamente reflejan el pensamiento de la Revista Ciencia Digital.

El artículo queda en propiedad de la revista y, por tanto, su publicación parcial y/o total en otro medio tiene que ser autorizado por el director de la Revista Ciencia Digital.

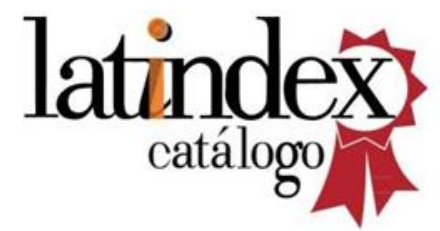

